\title{
Controllability and Reachability Criteria for Switched Linear Systems
}

\author{
Z. Sun, S. S. Ge* and T. H. Lee \\ Department of Electrical and Computer Engineering \\ National University of Singapore \\ Singapore 117576
}

\begin{abstract}
This paper investigates the controllability and reachability of switched linear control systems. It is proven that both the controllable and reachable sets are subspaces of the total space. Complete geometric characterization for both sets is presented. The switching control design problem is also addressed.
\end{abstract}

Keywords: Switched linear systems; Controllability; Reachability; Switching control

\section{Introduction}

During the last decade, hybrid and switched systems have attracted considerable attention (Chase, Serrano \& Ramadge 1993, Branicky 1998, Wicks, Peleties \& DeCarlo 1998, Ye, Michel \& Hou 1998, Liberzon \& Morse 1999). Basically, a switched system consists of continuous-time/discrete-time dynamical subsystems and a rule (supervisor) that determines the switching among them.

Switched systems deserve investigation for theoretical reasons as well as for practical reasons. Switching among different system structures is an essential feature of many engineering control applications including power systems and power electronics (Williams \& Hoft 1991, Sira-Ramirez 1991), and switched systems have numerous applications in control of mechanical systems, air traffic control, aircrafts and satellites and many other fields (Li, Wen \& Soh 2001). Control techniques by switching among different controllers have been applied extensively in recent years. Indeed, a switched controller can provide a performance improvement over a fixed controller (Morse 1996, Narendra \& Balakrishnan 1997, Savkin, Skafidas \& Evans 1999). The switched controller architecture is proven to be a rigorous design framework for general nonlinear systems (Kolmanovsky \& McClamroch 1996, Caines \& Wei 1998, Leonessa, Haddad \& Chellaboina 2001). A switched controller can also achieve certain control objects which

${ }^{*}$ To whom all correspondence should be addressed. Tel. (+65) 8746821; Fax. (+65) 7791103; E-mail: elegesz@nus.edu.sg 
cannot be accomplished by conventional methods, such as pure feedback stabilization of nonholonomic systems (Brockett 1983, Kolmanovsky \& McClamroch 1995).

A fundamental pre-requisite for the design of feedback control systems is full knowledge about the structural properties of the switched systems under consideration. These properties are closely related to the concepts of controllability, observability and stability which are of fundamental importance in the literature of control. There have been a lot of studies for switched systems, primarily on stability analysis and design (Branicky 1998, Dayawansa \& Martin 1999, Liberzon \& Morse 1999). As for controllability and reachability, studies for low-order switched linear systems have been presented in Loparo, Aslanis \& IIajek (1987) and Xu \& Antsaklis (1999). Some sufficient conditions and necessary conditions for controllability were presented in Ezzine \& Haddad (1989) and Szigeti (1992) for switched linear control systems under the assumption that the switching sequence is fixed a priori. The complexity of stability and controllability of hybrid systems was addressed in Blondel \& Tsitsiklis (1999).

For controllability analysis of switched linear control systems, a much more difficult situation arises since both the control input and the switching rule are design variables to be determined, and thus the interaction between them must be fully understood. For a switched linear discrete-time control system, the controllable set is not a subspace but a countable union of subspaces in general case (Stanford \& Conner 1980, Conner \& Stanford 1987). For a switched linear continuous-time control system, the controllable set is an uncountable union of subspaces (Sun \& Zheng 2001).

In this paper, we investigate the controllability and reachability issues for switched linear control systems in detail. We prove that, both the controllable set and the reachable set are subspaces of the total space, and the two sets always coincide with each other. Verifiable geometric characterization is presented for the controllable subspace. Dualistic criteria for observability and determinability are also presented.

The paper is organized as follows. In Section 2, we present the definitions of controllable and reachable notions. Preliminary results are given in Section 3. A complete characterization for the controllability and reachability sets is presented in Section 4 . In Section 5, we briefly address the observability and determinability issues. An illustrative example is presented in Section 6. Finally, some concluding remarks are made in Section 7. 


\section{Definitions}

Consider a switched linear control system given by

$$
\dot{x}(t)=A_{\sigma} x(t)+B_{\sigma} u_{\sigma}(t)
$$

where $x \in \Re^{n}$ are the states, $u_{k}: \Re^{+} \in \Re^{r_{k}}, k=1, \cdots, m$ are piecewise continuous input functions, $\sigma:\left[t_{0}, \infty\right) \rightarrow M=\{1,2, \cdots, m\}$ is the switching path to be designed, and matrix pairs $\left(A_{k}, B_{k}\right)$ for $k \in M$ are referred to as the subsystems of (1).

Given a switching path $\sigma:\left[t_{0}, t_{f}\right] \rightarrow M$, suppose its discontinuous (jump) time instants are $t_{1}<t_{2}<\cdots<t_{s}$, we refer to the sequence $t_{0}, t_{1}, t_{2}, \cdots, t_{s}$ as switching time sequence, and the sequence $\sigma\left(t_{0}\right), \sigma\left(t_{1}\right), \cdots, \sigma\left(t_{s}\right)$ as switching index sequence. It is clear that these two sequences can uniquely determine the switching path, and viceversa.

For clarity, let $x\left(t ; t_{0}, x_{0}, u, \sigma\right)$ denote the state trajectory at time $t$ of switched system (1) starting from $x\left(t_{0}\right)=x_{0}$ with $u(t)=\left[u_{1}(t), \cdots, u_{m}(t)\right]^{T}$.

A state $x$ is said to be controllable at time $t_{0}$, if it can be transferred to the origin in a finite time starting from $t_{0}$ by appropriate choices of input $u$ and switching path $\sigma$.

Definition 1. State $x \in \Re^{n}$ is controllable at time $t_{0}$, if there exist a time instant $t_{f}>t_{0}$, a switching path $\sigma:\left[t_{0}, t_{f}\right] \rightarrow M$, and inputs $u_{k}:\left[t_{0}, t_{f}\right] \rightarrow \Re^{r_{k}}, k \in M$, such that $x\left(t_{f} ; t_{0}, x, u, \sigma\right)=0$.

Definition 2. The controllable set of system (1) at $t_{0}$ is the set of states which are controllable at $t_{0}$.

Definition 3. System (1) is said to be (completely) controllable at time $t_{0}$, if its controllable set at $t_{0}$ is $\Re^{n}$.

The reachability counterparts can be defined in the same fashion as follows.

Definition 4. State $x \in \Re^{n}$ is reachable at $t_{0}$, if there exist a time instant $t_{f}>t_{0}$, a switching path $\sigma:\left[t_{0}, t_{f}\right] \rightarrow M$, and inputs $u_{k}:\left[t_{0}, t_{f}\right] \rightarrow \Re^{r_{k}}, k \in M$, such that $x\left(t_{f} ; t_{0}, 0, u, \sigma\right)=x$.

Definition 5. The reachable set of system (1) at $t_{0}$ is the set of states which are reachable at $t_{0}$.

Definition 6. System (1) is said to be (completely) reachable at $t_{0}$, if its reachable set at $t_{0}$ is $\Re^{n}$. 
Note that $x\left(t ; t_{0}, x_{0}, u, \sigma\right)=x\left(t^{\prime} ; t_{0}^{\prime}, x_{0}, u^{\prime}, \sigma^{\prime}\right)$ if $t^{\prime}-t=t_{0}^{\prime}-t_{0}, u^{\prime}(t)=u\left(t-t_{0}+t_{0}^{\prime}\right)$ and $\sigma^{\prime}(t)=\sigma\left(t-t_{0}+t_{0}^{\prime}\right)$ for all $t \in\left[t_{0}^{\prime}, t^{\prime}\right]$. That is, the state trajectory possesses the translation invariant property. Accordingly, if $x$ is controllable (reachable) at a time $t_{0}$, then $x$ is controllable (reachable) at any arbitrary given instant of time. In the sequel, the reference of $t_{0}$ shall be dropped for conciseness.

It is obvious that if one subsystem, say $\left(A_{1}, B_{1}\right)$, is controllable, then system (1) is both controllable and reachable. In this paper, we shall investigate the non-trivial situation where each subsystem $\left(A_{k}, B_{k}\right), k \in M$ is not controllable.

\section{$3 \quad$ Elementary results}

\subsection{Elementary analysis}

Given an initial state $x\left(t_{0}\right)=x_{0}$, inputs $u_{k}, k \in M$, and a switching path $\sigma:\left[t_{0}, t_{f}\right] \rightarrow$ $M$, the solution of state equation (1) is given by

$$
\begin{gathered}
x(t)=e^{A_{i_{k}}\left(t-t_{k}\right)} \cdots e^{A_{i_{0}}\left(t_{1}-t_{0}\right)} x_{0}+e^{A_{i_{k}}\left(t-t_{k}\right)} \cdots e^{A_{i_{1}}\left(t_{2}-t_{1}\right)} \int_{t_{0}}^{t_{1}} e^{A_{i_{0}}\left(t_{1}-\tau\right)} B_{i_{0}} u_{i_{0}}(\tau) d \tau \\
+\cdots+e^{A_{i_{k}}\left(t-t_{k}\right)} \int_{t_{k-1}}^{t_{k}} e^{A_{i_{k-1}}\left(t_{k}-\tau\right)} B_{i_{k-1}} u_{i_{k-1}}(\tau) d \tau+\int_{t_{k}}^{t} e^{A_{i_{k}}(t-\tau)} B_{i_{k}} u_{i_{k}}(\tau) d \tau \\
\quad \text { for } t_{k}<t \leq t_{k+1}, \quad 1 \leq k \leq s
\end{gathered}
$$

where $t_{0}, t_{1}, \cdots, t_{s}$ is the switching time sequence of $\sigma, t_{s+1}=t_{f}$, and $i_{0}=\sigma\left(t_{0}\right), \cdots$, $i_{s}=\sigma\left(t_{s}\right)$ is the switching index sequence of $\sigma$.

The reachable set of system (1) is given by

$$
\begin{aligned}
\mathcal{R}=\{ & \left.x: x=x\left(t ; t_{0}, 0, u, \sigma\right) \text { with } t \geq t_{0}, u \in U^{r}, \text { and } \sigma:\left[t_{0}, t\right] \rightarrow M\right\} \\
=\{ & x: x=e^{A_{i_{k}}\left(t-t_{k}\right)} \cdots e^{A_{i_{1}}\left(t_{2}-t_{1}\right)} \int_{t_{0}}^{t_{1}} e^{A_{i_{0}}\left(t_{1}-\tau\right)} B_{i_{0}} u_{i_{0}}(\tau) d \tau+\cdots \\
& +e^{A_{i_{k}}\left(t-t_{k}\right)} \int_{t_{k-1}}^{t_{k}} e^{A_{i_{k-1}}\left(t_{k}-\tau\right)} B_{i_{k-1}} u_{i_{k-1}}(\tau) d \tau+\int_{t_{k}}^{t} e^{A_{i_{k}}(t-\tau)} B_{i_{k}} u_{i_{k}}(\tau) d \tau, \\
& \text { for } \left.k \geq 1, t_{0}<t_{1}<\cdots<t_{k}<t, i_{j} \in M, j=0, \cdots, k, \text { and } u \in U^{r}\right\}
\end{aligned}
$$

where $r=\sum_{k=1}^{m} r_{k}$ and $U^{r}$ is the set of $r$ th-dimensional piecewise continuous vector functions. 
Note that for any matrices $A \in \Re^{n \times n}, B \in \Re^{n \times p}$ and $t>t_{0}$, we have

$$
\left\{x: x=\int_{t_{0}}^{t} e^{A(t-\tau)} B u(\tau) d \tau \text { with } u \in U^{p}\right\}=\sum_{k=0}^{n-1} A^{k} \operatorname{Im} B
$$

where $\operatorname{Im} B$ is the subspace spanned by columns of matrix $B$.

Denote $D_{k}=\left[B_{k}, A_{k} B_{k}, \cdots, A_{k}^{n-1} B_{k}\right], \mathcal{B}_{k}=\operatorname{Im} B_{k}$, and $\mathcal{D}_{k}=\operatorname{Im} D_{k}$ for $k \in M$. It follows from (3) that the reachable set

$$
\mathcal{R}=\cup_{k=1}^{\infty} \cup_{i_{0}, \cdots, i_{k} \in M} \cup_{h_{1}, \cdots, h_{k}>0}\left(e^{A_{i_{k}} h_{k}} \cdots e^{A_{i_{1}} h_{1}} \mathcal{D}_{i_{0}}+\cdots+e^{A_{i_{k}} h_{k}} \mathcal{D}_{i_{k-1}}+\mathcal{D}_{i_{k}}\right)
$$

Similarly, the controllable set of system (1) is given by

$$
\mathcal{C}=\cup_{k=1}^{\infty} \cup_{i_{0}, \cdots, i_{k} \in M} \cup_{h_{0}, \cdots, h_{k}>0}\left(e^{-A_{i_{0}} h_{0}} \mathcal{D}_{i_{0}}+\cdots+e^{-A_{i_{0}} h_{0}} \cdots e^{-A_{i_{k}} h_{k}} \mathcal{D}_{i_{k}}\right)
$$

Given a matrix $A$ and a subspace $\mathcal{B} \in \Re^{n}$, let $\Gamma_{A} \mathcal{B}$ denote the minimal $A$-invariant subspace that contains $\mathcal{B}$, i.e.,

$$
\Gamma_{A} \mathcal{B}=\mathcal{B}+A \mathcal{B}+\cdots+A^{n-1} \mathcal{B}
$$

This operation can be defined recursively as $\Gamma_{A_{1}} \Gamma_{A_{2}} \mathcal{B}=\Gamma_{A_{1}}\left(\Gamma_{A_{2}} \mathcal{B}\right)$. Let us define the nested subspaces as

$$
\begin{aligned}
\mathcal{V}_{1} & =\mathcal{D}_{1}+\cdots+\mathcal{D}_{m} \\
\mathcal{V}_{j+1} & =\Gamma_{A_{1}} \mathcal{V}_{j}+\cdots+\Gamma_{A_{m}} \mathcal{V}_{j}, \quad j=1,2, \cdots
\end{aligned}
$$

and

$$
\mathcal{V}=\sum_{k=1}^{\infty} \mathcal{V}_{k}
$$

Note that if $\operatorname{dim} \mathcal{V}_{j}=\operatorname{dim} \mathcal{V}_{j+1}$, then $\mathcal{V}_{l}=\mathcal{V}_{j}$ for $l>j$. This fact implies that $\mathcal{V}=\mathcal{V}_{n}$. It is readily seen that this subspace is the minimal subspace which is invariant under $A_{k}$, $k \in M$ and contains $\sum_{k \in M} \mathcal{B}_{k}$. Subspace $\mathcal{V}$ plays an important role in the following derivations.

Note that $e^{A t} \operatorname{Im} B \subset \Gamma_{A} \operatorname{Im} B$ for all $A \in \Re^{n \times n}, B \in \Re^{n \times p}$ and $t \in \Re$. This gives

$$
\mathcal{R} \subset \cup_{k=1}^{\infty} \cup_{i_{0}, \cdots, i_{k} \in M}\left(\Gamma_{A_{i_{k}}} \cdots \Gamma_{A_{i_{1}}} \mathcal{D}_{i_{0}}+\cdots+\mathcal{D}_{i_{k}}\right) \subset \mathcal{V}
$$

and

$$
\mathcal{C} \subset \cup_{k=1}^{\infty} \cup_{i_{0}, \cdots, i_{k} \in M}\left(\mathcal{D}_{i_{0}}+\cdots+\Gamma_{A_{i_{0}}} \cdots \Gamma_{A_{i_{k-1}}} \mathcal{D}_{i_{k}}\right) \subset \mathcal{V}
$$

As has been shown in Sun \& Zheng (2001), we have the following proposition. 
Proposition 1. If switched linear system (1) is controllable or reachable, then

$$
\mathcal{V}=\Re^{n}
$$

\subsection{A heuristic example}

According to (4) and (5), the controllable set and the reachable set of system (1) are uncountable unions of subspaces of $\Re^{n}$. A question arises naturally: Are $\mathcal{R}$ and $\mathcal{C}$ subspaces of $\Re^{n}$ ? A heuristic way for addressing this question is by analyzing typical examples.

Example 1. Consider system (1) with $n=4, m=2$, and

$$
A_{1}=\left[\begin{array}{llll}
0 & 0 & 0 & 0 \\
0 & 0 & 0 & 0 \\
0 & 1 & 0 & 0 \\
0 & 0 & 0 & 0
\end{array}\right], B_{1}=\left[\begin{array}{l}
1 \\
0 \\
0 \\
0
\end{array}\right] ; A_{2}=\left[\begin{array}{cccc}
0 & 0 & 0 & 0 \\
1 & 0 & 0 & 0 \\
0 & 0 & 0 & 0 \\
0 & 0 & 0 & 0
\end{array}\right], B_{2}=\left[\begin{array}{l}
0 \\
0 \\
0 \\
0
\end{array}\right]
$$

Simple calculation gives

$$
\mathcal{V}=\operatorname{span}\left\{\left[\begin{array}{l}
1 \\
0 \\
0 \\
0
\end{array}\right],\left[\begin{array}{l}
0 \\
1 \\
0 \\
0
\end{array}\right],\left[\begin{array}{l}
0 \\
0 \\
1 \\
0
\end{array}\right]\right\}
$$

It follows from Proposition 1 that system (10) is neither controllable nor reachable.

Now we compute the reachable set for system (10). For clarity, let $\mathcal{R}_{j}$ denote the set of points which can be transfered from the origin within $j$ times of switching. Accordingly,

$$
\begin{aligned}
& \mathcal{R}_{0}=\mathcal{D}_{1} \cup \mathcal{D}_{2}=\operatorname{span}\left\{\left[\begin{array}{l}
1 \\
0 \\
0 \\
0
\end{array}\right]\right\} \\
& \mathcal{R}_{1}=\left(\cup_{t \geq 0} e^{A_{2} t} \mathcal{R}_{0}+\Gamma_{A_{2}} \mathcal{B}_{2}\right) \cup\left(\cup_{t \geq 0} e^{A_{1} t} \mathcal{R}_{0}+\Gamma_{A_{1}} \mathcal{B}_{1}\right)=\left\{\left[\begin{array}{c}
a \\
a t \\
0 \\
0
\end{array}\right]: a \in \Re, t \geq 0\right\}
\end{aligned}
$$


Note that set $\mathcal{R}_{1}$ is neither a subspace nor a countable unions of subspaces.

Further calculation yields

$$
\begin{aligned}
\mathcal{R}_{2}= & \left(\cup_{t \geq 0} e^{A_{2} t} \mathcal{R}_{1}+\Gamma_{A_{2}} \mathcal{B}_{2}\right) \cup\left(\cup_{t \geq 0} e^{A_{1} t} \mathcal{R}_{1}+\Gamma_{A_{1}} \mathcal{B}_{1}\right)=\left\{\left[\begin{array}{c}
a \\
b \\
b t \\
0
\end{array}\right]: a, b \in \Re, t \geq 0\right\} \\
\mathcal{R}_{3}= & \left(\cup_{t \geq 0} e^{A_{2} t} \mathcal{R}_{2}+\Gamma_{A_{2}} \mathcal{B}_{2}\right) \cup\left(\cup_{t \geq 0} e^{A_{1} t} \mathcal{R}_{2}+\Gamma_{A_{1}} \mathcal{B}_{1}\right) \\
& =\left\{\left[\begin{array}{c}
a \\
a t_{3}+b \\
b t_{2} \\
0
\end{array}\right]: a, b \in \Re, t_{2}, t_{3} \geq 0\right\}
\end{aligned}
$$

Sets $\mathcal{R}_{2}$ and $\mathcal{R}_{3}$ are strict subsets of $\mathcal{V}$, and $\mathcal{R}_{3}$ strictly include $\mathcal{R}_{2}$ as a subset.

Repeating this process, we have

$$
\begin{aligned}
\mathcal{R}_{4} & =\left(\cup_{t \geq 0} e^{A_{2} t} \mathcal{R}_{3}+\Gamma_{A_{2}} \mathcal{B}_{2}\right) \cup\left(\cup_{t \geq 0} e^{A_{1} t} \mathcal{R}_{3}+\Gamma_{A_{1}} \mathcal{B}_{1}\right) \\
& =\left\{\left[\begin{array}{c}
a \\
b+c t_{3} \\
b t_{2} \\
0
\end{array}\right]: a, b, c \in \Re, t_{2}, t_{3} \geq 0\right\}=\operatorname{span}\left\{\left[\begin{array}{l}
1 \\
0 \\
0 \\
0
\end{array}\right],\left[\begin{array}{l}
0 \\
1 \\
0 \\
0
\end{array}\right],\left[\begin{array}{l}
0 \\
0 \\
1 \\
0
\end{array}\right]\right\}=\mathcal{V}
\end{aligned}
$$

From (7), it follows that the reachable set of system (10) is exactly $\mathcal{V}$, which is a subspace of $\Re^{4}$. 
By analogy, the controllable counterparts are given by

$$
\begin{aligned}
& \mathcal{C}_{0}=\mathcal{D}_{1} \cup \mathcal{D}_{2}=\operatorname{span}\left\{\left[\begin{array}{l}
1 \\
0 \\
0 \\
0
\end{array}\right]\right\} \\
& \mathcal{C}_{1}=\left\{\left[\begin{array}{c}
a \\
-a t \\
0 \\
0
\end{array}\right]: a \in \Re, t \geq 0\right\} \\
& \mathcal{C}_{2}=\left\{\left[\begin{array}{c}
a \\
b \\
-b t \\
0
\end{array}\right]: a, b \in \Re, t \geq 0\right\} \\
& \mathcal{C}_{3}=\left\{\left[\begin{array}{c}
a \\
-a t_{2}+b \\
-b t_{1} \\
0
\end{array}\right]: a, b \in \Re, t_{1}, t_{2} \geq 0\right\}
\end{aligned}
$$

and

$$
\mathcal{C}_{4}=\left\{\left[\begin{array}{c}
a \\
-a t_{3}+b-c t_{2} \\
-b t_{1} \\
0
\end{array}\right]: a, b, c \in \Re, t_{1}, t_{2}, t_{3} \geq 0\right\}=\mathcal{V}
$$

From (8), it follows that the controllable set of system (10) is exactly $\mathcal{V}$, which is a subspace of $\Re^{4}$.

To summarize, for system (10), we have

(i) Both the controllable set and the reachable set are subspaces.

(ii) $\mathcal{R}=\mathcal{C}=\mathcal{V}$.

(iii) Not all $\mathcal{R}_{j}$ and $\mathcal{C}_{j}$ are subspaces, and $\mathcal{R}_{j} \neq \mathcal{C}_{j}$ for $j=1,2,3$.

(iv) The dimension of $\mathcal{C}$ is three, while it needs four times of switching to transfer an arbitrary any given configuration in $\mathcal{C}$ to the origin. 
Properties (i) and (ii) are parallel to the non-switching case while properties (iii) and (iv) indicate complex phenomena arising when switching between different subsystems occurs.

\subsection{Rank divergent properties of $e^{A t}$}

As expressed in (2), the state transition matrix for switched system (1) is multiple multiplication of matrix function of the form $e^{A t}$. Accordingly, properties of exponential matrix functions play an important role in structural analysis for switched linear systems. In this subsection, several good properties for $e^{A t}$ (called rank divergent properties for convenience) shall be presented. These properties are crucial to the derivations of the main results.

Lemma 1. For any given matrix $A \in \Re^{n \times n}$ and subspace $\mathcal{B} \subset \Re^{n}$, the following equation holds for almost all $t_{1}, t_{2}, \cdots, t_{n} \in \Re$

$$
e^{A t_{1}} \mathcal{B}+e^{A t_{2}} \mathcal{B}+\cdots+e^{A t_{n}} \mathcal{B}=\Gamma_{A} \mathcal{B}
$$

Proof. Let $\mathcal{S}$ be the smallest subspace of $\Re^{n}$ that contains the subspaces $e^{A t} \mathcal{B}$ for all $t \in \Re$. That is, $\mathcal{S}$ is spanned by the set of vectors

$$
\left\{e^{A t} B z: t \in \Re, z \in \Re^{n}\right\}
$$

By (Drager, Foote, Martin \& Wolfer 1989, Proposition 2.1), $\mathcal{S}$ is exactly the controllable subspace of matrix pair $(A, B)$ :

$$
\mathcal{S}=\operatorname{span}\left\{e^{A t} B z: t \in \Re, z \in \Re^{n}\right\}=\Gamma_{A} \mathcal{B}
$$

Suppose $e^{A t_{j}^{0}} B z_{j}, \quad j=1, \cdots, n$ spans subspace $\mathcal{S}$, i.e.,

$$
\mathcal{S}=\operatorname{span}\left\{e^{A t_{1}^{0}} B z_{1}, \cdots, e^{A t_{n}^{0}} B z_{n}\right\}
$$

This implies that

$$
e^{A t_{1}^{0}} \mathcal{B}+\cdots+e^{A t_{n}^{0}} \mathcal{B}=\Gamma_{A} \mathcal{B}
$$

or equivalently,

$$
\operatorname{rank}\left[e^{A t_{1}^{0}} B, \cdots, e^{A t_{n}^{0}} B\right]=\operatorname{dim}\left(\Gamma_{A} \mathcal{B}\right)
$$


Denote integer $r=\operatorname{dim}\left(\Gamma_{A} \mathcal{B}\right)$, and matrix function $L\left(t_{1}, \cdots, t_{n}\right)=\left[e^{A t_{1}} B, \cdots, e^{A t_{n}} B\right]$. Choose a nonsingular sub-matrix $M_{0}$ with maximal rank in $L\left(t_{1}^{0}, \cdots, t_{n}^{0}\right)$. Therefore, $M_{0}$ is nonsingular and $\operatorname{rank} M_{0}=\operatorname{rank} L\left(t_{1}^{0}, \cdots, t_{n}^{0}\right)$. Denote the corresponding submatrix of $L\left(t_{1}, \cdots, t_{n}\right)$ as $M\left(t_{1}, \cdots, t_{n}\right)$, and its determinant as $d\left(t_{1}, \cdots, t_{n}\right)$.

Since each entry in matrix $M\left(t_{1}, \cdots, t_{n}\right)$ is an analytic function of variables $t_{1}, \cdots, t_{n}$, $d\left(t_{1}, \cdots, t_{n}\right)$ is also an analytic function of its variables. As $d\left(t_{1}^{0}, \cdots, t_{n}^{0}\right) \neq 0$, function $d\left(t_{1}, \cdots, t_{n}\right)$ is not identically zero. By Weierstrass Preparation Theorem (Kaplan 1966, Theorem 62), its zeros forms a zero-measure set of $\Re^{n}$. Therefore, for almost all $t_{1}, \cdots, t_{n}$, matrix $M\left(t_{1}, \cdots, t_{n}\right)$ is nonsingular. This implies that

$$
\operatorname{rank} L\left(t_{1}, \cdots, t_{n}\right) \geq \operatorname{rank} M\left(t_{1}, \cdots, t_{n}\right)=r=\operatorname{dim}\left(\Gamma_{A} \mathcal{B}\right)
$$

for almost all $t_{1}, \cdots, t_{n}$. Together with the fact that $\mathcal{S} \subseteq \Gamma_{A} \mathcal{B}$, we can conclude that

$$
e^{A t_{1}} \mathcal{B}+\cdots+e^{A t_{n}} \mathcal{B}=\Gamma_{A} \mathcal{B}
$$

for almost all $t_{1}, \cdots, t_{n} . \diamond$

Lemma 2. For any given matrices $A_{k} \in \Re^{n \times n}$ and $B_{k} \in \Re^{n \times p_{k}}, k=1,2$, inequality

$$
\operatorname{rank}\left[A_{1} e^{A_{2} t} B_{1}, B_{2}\right] \geq \operatorname{rank}\left[A_{1} B_{1}, B_{2}\right]
$$

holds for almost all $t \in \Re$.

Proof. Denote matrix function $\Omega(t)=\left[A_{1} e^{A_{2} t} B_{1}, B_{2}\right]$. Choose a nonsingular sub-matrix $G$ with maximal rank in $\Omega(0)=\left[A_{1} B_{1}, B_{2}\right]$. Denote the corresponding sub-matrix of $\Omega(t)$ as $\Delta(t)$, and its determinant as $\delta(t)$. It is standard that all elements of $\Delta(t)$ are linear combinations of the form $t^{k} e^{\lambda t}$, hence $\delta: \Re \rightarrow \Re$ is an analytic function on $\Re$. Because $\delta(0)=\operatorname{det} G \neq 0$, the zeros of $\delta(t)$ are isolated points (Kaplan 1966, Theorem 43). Consequently, $\delta(t) \neq 0$ for almost all $t \in \Re$. Accordingly, for almost all $t, \Delta(t)$ is nonsingular. Therefore,

$$
\operatorname{rank} \Omega(t) \geq \operatorname{rank} \Delta(t)=\operatorname{rank} G=\operatorname{rank}\left[A_{1} B_{1}, B_{2}\right]
$$

for almost all $t . \diamond$

Note that inequality (13) cannot be substituted by equality as shown by the following example

$$
\operatorname{rank}\left[e^{A t} b, b\right]>\operatorname{rank}[b, b] \text { for } A_{1}=I_{3}, A=\left[\begin{array}{ccc}
0 & 1 & 0 \\
0 & 0 & 1 \\
0 & 0 & 0
\end{array}\right], b=\left[\begin{array}{l}
0 \\
0 \\
1
\end{array}\right], t \neq 0
$$




\section{Main results}

\subsection{Geometric criteria}

In this subsection, we shall identify the controllable set and the reachable set for switched linear systems.

Theorem 1. For switched linear system (1), the reachable set is

$$
\mathcal{R}=\mathcal{V}
$$

Proof. We are to design a switching path $\sigma$ such that each point in $\mathcal{V}$ can be reached from the origin via this switching path.

Assume that the switching index sequence of $\sigma$ is periodic. i.e.,

$$
\begin{aligned}
& i_{0}=1, \quad i_{1}=2, \quad \cdots, \quad i_{m-1}=m, \\
& i_{m}=1, \quad i_{m+1}=2, \quad \cdots, \quad i_{2 m-1}=m, \quad \cdots
\end{aligned}
$$

The switching time sequence $t_{0}, \cdots, t_{l}$ and the number $l$ are to be designed later.

Let $t_{f}>t_{l}$. From (4), the reachable set at $t_{f}$ is

$$
\mathcal{R}\left(t_{f}\right)=e^{A_{i_{l}} h_{l}} \cdots e^{A_{2} h_{1}} \mathcal{D}_{1}+\cdots+e^{A_{i_{l}} h_{l}} \mathcal{D}_{i_{l-1}}+\mathcal{D}_{i_{l}}
$$

where $h_{j}=t_{j+1}-t_{j}, j=0,1, \cdots, l-1$ and $h_{l}=t_{f}-t_{l}$.

Since

$$
\begin{aligned}
& e^{A_{i_{l}} h_{l}} \cdots e^{A_{2} h_{1}} \mathcal{D}_{1}+\cdots+e^{A_{i_{l}} h_{l}} \mathcal{D}_{i_{l-1}}+\mathcal{D}_{i_{l}} \\
& =e^{A_{i_{l}} h_{l}}\left(e^{A_{i_{l-1}} h_{l-1}} \cdots e^{A_{2} h_{1}} \mathcal{D}_{1}+\cdots+e^{A_{i_{l-1}} h_{l-1}} \mathcal{D}_{i_{l-2}}+\mathcal{D}_{i_{l-1}}\right)+\mathcal{D}_{i_{l}}
\end{aligned}
$$

it follows from Lemma 2 that

$$
\begin{aligned}
& \operatorname{dim}\left(e^{A_{i_{l}} h_{l}} \cdots e^{A_{2} h_{1}} \mathcal{D}_{1}+\cdots+e^{A_{i_{l}} h_{l}} \mathcal{D}_{i_{l-1}}+\mathcal{D}_{i_{l}}\right) \\
& \geq \operatorname{dim}\left(e^{A_{i_{l-1}} h_{l-1}} \cdots e^{A_{2} h_{1}} \mathcal{D}_{1}+\cdots+e^{A_{i_{l-1}} h_{l-1}} \mathcal{D}_{i_{l-2}}+\mathcal{D}_{i_{l-1}}+\mathcal{D}_{i_{l}}\right)
\end{aligned}
$$

for almost all $h_{l}$. 
By repeatedly applying Lemma 2 , for almost all $h_{l}, \cdots, h_{l-m+1}$, we have

$$
\begin{aligned}
& \operatorname{dim}\left(e^{A_{i_{l}} h_{l}} \cdots e^{A_{2} h_{1}} \mathcal{D}_{1}+\cdots+e^{A_{i_{l}} h_{l}} \mathcal{D}_{i_{l-1}}+\mathcal{D}_{i_{l}}\right) \\
& \geq \operatorname{dim}\left(e^{A_{i_{l-1}} h_{l-1}} \cdots e^{A_{2} h_{1}} \mathcal{D}_{1}+\cdots+e^{A_{i_{l-1}} h_{l-1}} \mathcal{D}_{i_{l-2}}+\mathcal{D}_{i_{l-1}}+\mathcal{D}_{i_{l}}\right) \\
& \vdots \\
& \geq \operatorname{dim}\left(e^{A_{i_{\tau_{1}}} h_{\tau_{1}} \cdots e^{A_{2} h_{1}}} \mathcal{D}_{1}+\cdots+e^{A_{i_{1}} h_{\tau_{1}}} \mathcal{D}_{i_{\tau_{1}-1}}+\mathcal{D}_{i_{\tau_{1}}}+\cdots+\mathcal{C}_{i_{l}}\right) \\
& =\operatorname{dim}\left(e^{A_{i_{1}} h_{\tau_{1}}} \cdots e^{A_{2} h_{1}} \mathcal{D}_{1}+\cdots+\mathcal{D}_{i_{\tau_{1}}}+\mathcal{V}_{1}\right)
\end{aligned}
$$

where $\tau_{1}=l-m$.

It follows from Lemma 2 that

$$
\begin{aligned}
& \operatorname{dim}\left(e^{A_{i_{1}} h_{\tau_{1}}} \cdots e^{A_{2} h_{1}} \mathcal{D}_{1}+\cdots+\mathcal{D}_{i_{\tau_{1}}}+\mathcal{V}_{1}\right) \\
& =\operatorname{dim}\left(e ^ { A _ { i _ { 1 } } h _ { \tau _ { 1 } } } e ^ { A _ { i _ { \tau _ { 1 } - 1 } } h _ { \tau _ { 1 } - 1 } } \left(e^{A_{\tau_{\tau_{1}}-2} h_{\tau_{1}-2}} \cdots e^{A_{2} h_{1}} \mathcal{D}_{1}+\cdots+e^{A_{i_{\tau_{1}-2}} h_{\tau_{1}-2}} \mathcal{D}_{\tau_{1}-3}\right.\right. \\
& \left.\left.\quad+\mathcal{D}_{\tau_{1}-2}\right)+e^{A_{i_{\tau_{1}}} h_{\tau_{1}}} \mathcal{D}_{i_{\tau_{1}-1}}+\mathcal{D}_{i_{\tau_{1}}}+\mathcal{V}_{1}\right) \\
& \geq \operatorname{dim}\left(e^{A_{i_{\tau_{1}}} h_{\tau_{1}}}\left(e^{A_{i_{\tau_{1}-2}} h_{\tau_{1}-2}} \cdots e^{A_{2} h_{1}} \mathcal{D}_{1}+\cdots+e^{A_{i_{\tau_{1}-2}} h_{\tau_{1}-2}} \mathcal{D}_{\tau_{1}-3}+\mathcal{D}_{\tau_{1}-2}\right)\right. \\
& \left.\quad+e^{A_{i_{\tau_{1}}} h_{\tau_{1}}} \mathcal{D}_{i_{\tau_{1}-1}}+\mathcal{D}_{i_{\tau_{1}}}+\mathcal{V}_{1}\right) \\
& =\operatorname{dim}\left(e^{A_{i_{\tau_{1}}} h_{\tau_{1}}}\left(e^{A_{\tau_{\tau_{1}-2}} h_{\tau_{1}-2}} \cdots e^{A_{2} h_{1}} \mathcal{D}_{1}+\cdots+e^{A_{\tau_{\tau_{1}-2}} h_{\tau_{1}-2}} \mathcal{D}_{\tau_{1}-3}+\mathcal{D}_{\tau_{1}-2}+\mathcal{D}_{i_{\tau_{1}-1}}\right)\right. \\
& \left.\quad+\mathcal{D}_{i_{\tau_{1}}}+\mathcal{V}_{1}\right)
\end{aligned}
$$

for almost all $h_{\tau_{1}-1}$.

By the same reasonings, we have

$$
\begin{aligned}
& \operatorname{dim}\left(e^{A_{i_{1}} h_{\tau_{1}}} \cdots e^{A_{2} h_{1}} \mathcal{D}_{1}+\cdots+\mathcal{D}_{i_{\tau_{1}}}+\mathcal{V}_{1}\right) \\
& \geq \operatorname{dim}\left(e ^ { A _ { i _ { 1 } } h _ { \tau _ { 1 } } } \left(e^{A_{i_{1}-2} h_{\tau_{1}-2}} \cdots e^{A_{2} h_{1}} \mathcal{D}_{1}+\cdots+e^{A_{\tau_{1}-2} h_{\tau_{1}-2}} \mathcal{D}_{\tau_{1}-3}\right.\right. \\
& \left.\left.+\mathcal{D}_{\tau_{1}-2}+\mathcal{D}_{i_{\tau_{1}-1}}\right)+\mathcal{D}_{i_{\tau_{1}}}+\mathcal{V}_{1}\right) \\
& \geq \operatorname{dim}\left(e ^ { A _ { i _ { 1 } } h _ { \tau _ { 1 } } } \left(e^{A_{\tau_{\tau_{1}-3}} h_{\tau_{1}-3}} \cdots e^{A_{2} h_{1}} \mathcal{D}_{1}+\cdots+e^{A_{\tau_{\tau_{1}-3}} h_{\tau_{1}-3}} \mathcal{D}_{\tau_{1}-4}\right.\right. \\
& \left.\left.+\mathcal{D}_{\tau_{1}-3}+\cdots+\mathcal{D}_{i_{\tau_{1}-1}}\right)+\mathcal{D}_{i_{\tau_{1}}}+\mathcal{V}_{1}\right) \\
& \vdots \\
& \geq \operatorname{dim}\left(e ^ { A _ { i _ { 1 } } h _ { \tau _ { 1 } } } \left(e^{A_{i_{\tau_{1}-m}} h_{\tau_{1}-m}} \cdots e^{A_{2} h_{1}} \mathcal{D}_{1}+\cdots+e^{A_{i_{\tau_{1}}-m} h_{\tau_{1}-m}} \mathcal{D}_{\tau_{1}-m-1}\right.\right. \\
& \left.\left.+\mathcal{D}_{\tau_{1}-m}+\cdots+\mathcal{D}_{i_{\tau_{1}-1}}\right)+\mathcal{D}_{i_{\tau_{1}}}+\mathcal{V}_{1}\right) \\
& =\operatorname{dim}\left(e^{A_{\tau_{1}} h_{\tau_{1}}} e^{A_{i_{\tau_{1}-m}} h_{\tau_{1}-m}}\left(e^{A_{i_{\tau_{1}-m-1}} h_{\tau_{1}-m-1}} \cdots e^{A_{2} h_{1}} \mathcal{D}_{1}+\cdots+\mathcal{D}_{\tau_{1}-m-1}\right)\right. \\
& \left.+e^{A_{i_{\tau_{1}}} h_{\tau_{1}}} \mathcal{V}_{1}+\mathcal{D}_{i_{\tau_{1}}}+\mathcal{V}_{1}\right)
\end{aligned}
$$

for almost all $h_{j}, j=\tau_{1}-1, \cdots, \tau_{1}-m+1$. 
Continuing the above process gives

$$
\begin{aligned}
& \operatorname{dim}\left(e^{A_{i_{1}} h_{\tau_{1}}} \cdots e^{A_{2} h_{1}} \mathcal{D}_{1}+\cdots+\mathcal{D}_{i_{\tau_{1}}}+\mathcal{V}_{1}\right) \\
& \geq \operatorname{dim}\left(e^{A_{i_{1}} h_{\tau_{1}}} e^{A_{i_{\tau_{1}-m}} h_{\tau_{1}-m}}\left(e^{A_{i_{\tau_{1}-m-1}} h_{\tau_{1}-m-1}} \cdots e^{A_{2} h_{1}} \mathcal{D}_{1}+\cdots+\mathcal{D}_{\tau_{1}-m-1}\right)\right. \\
& \left.+e^{A_{i_{1}} h_{\tau_{1}}} \mathcal{V}_{1}+\mathcal{D}_{i_{\tau_{1}}}+\mathcal{V}_{1}\right) \\
& \geq \operatorname{dim}\left(e ^ { A _ { i _ { 1 } } h _ { \tau _ { 1 } } } e ^ { A _ { i _ { \tau _ { 1 } - m } } h _ { \tau _ { 1 } - m } } e ^ { A _ { i _ { \tau _ { 1 } - 2 m } } h _ { \tau _ { 1 } - 2 m } } \left(e^{A_{i_{\tau_{1}-2 m-1}} h_{\tau_{1}-2 m-1}} \cdots e^{A_{2} h_{1}} \mathcal{D}_{1}+\cdots\right.\right. \\
& \left.\left.+\mathcal{D}_{\tau_{1}-2 m-1}\right)+e^{A_{i_{1}} h_{\tau_{1}}} e^{A_{\tau_{\tau_{1}-m}} h_{\tau_{1}-m}} \mathcal{V}_{1}+e^{A_{i_{1}} h_{\tau_{1}}} \mathcal{V}_{1}+\mathcal{D}_{i_{\tau_{1}}}+\mathcal{V}_{1}\right) \\
& \vdots \\
& \geq \operatorname{dim}\left(e ^ { A _ { i _ { 1 } } h _ { \tau _ { 1 } } } e ^ { A _ { i _ { \tau _ { 1 } - m } } h _ { \tau _ { 1 } - m } } \cdots e ^ { A _ { i _ { \tau _ { 1 } - n m } } h _ { \tau _ { 1 } - n m } } \left(e^{A_{i_{\tau_{1}-n m-1}} h_{\tau_{1}-n m-1}} \cdots e^{A_{2} h_{1}} \mathcal{D}_{1}+\cdots\right.\right. \\
& \left.\left.+\mathcal{D}_{\tau_{1}-n m-1}\right)+e^{A_{i_{1}} h_{\tau_{1}}} \cdots e^{A_{i_{\tau_{1}-n m+m}} h_{\tau_{1}-n m+m}} \mathcal{V}_{1}+\cdots+e^{A_{i_{1}} h_{\tau_{1}}} \mathcal{V}_{1}+\mathcal{D}_{i_{\tau_{1}}}+\mathcal{V}_{1}\right) \\
& =\operatorname{dim}\left(e ^ { A _ { i _ { 1 } } h _ { \tau _ { 1 } } } e ^ { A _ { i _ { \tau _ { 1 } - m } } h _ { \tau _ { 1 } - m } } \cdots e ^ { A _ { i _ { \tau _ { 1 } - n m } } h _ { \tau _ { 1 } - n m } } \left(e^{A_{i_{\tau_{1}-n m-1}} h_{\tau_{1}-n m-1}} \cdots e^{A_{2} h_{1}} \mathcal{D}_{1}+\cdots\right.\right. \\
& \left.\left.+\mathcal{D}_{\tau_{1}-n m-1}\right)+e^{A_{i_{1}}\left(h_{\tau_{1}}+\cdots+h_{\tau_{1}-n m+m}\right)} \mathcal{V}_{1}+\cdots+e^{A_{i_{\tau_{1}}} h_{\tau_{1}}} \mathcal{V}_{1}+\mathcal{D}_{i_{\tau_{1}}}+\mathcal{V}_{1}\right)
\end{aligned}
$$

for almost all $h_{j}, j=\tau_{1}-m n+1, \cdots, \tau_{1}-m n+m-1, \tau_{1}-m n+m+1, \cdots, \tau_{1}-$ $m n+2 m-1, \cdots, \tau_{1}-m+1, \cdots, \tau_{1}-1$. The relationships $i_{j}=i_{j+m}, j=1,2, \cdots$ have been used in the last equation.

From Lemma 1, we have

$$
e^{A_{i_{1}}\left(h_{\tau_{1}}+h_{\tau_{1}-m}+\cdots+h_{\tau_{1}-m n+m}\right)} \mathcal{V}_{1}+\cdots+e^{A_{i_{1}} h_{\tau_{1}}} \mathcal{V}_{1}=\Gamma_{A_{i_{1}}} \mathcal{V}_{1}
$$

for almost all $h_{j}, j=\tau_{1}, \tau_{1}-m, \cdots, \tau_{1}-m n$. Accordingly, we can rewrite (18) as

$$
\begin{aligned}
& \operatorname{dim}\left(e^{A_{\tau_{1}} h_{\tau_{1}}} \cdots e^{A_{2} h_{1}} \mathcal{D}_{1}+\cdots+\mathcal{D}_{i_{\tau_{1}}}+\mathcal{V}_{1}\right) \\
& \geq \operatorname{dim}\left(e ^ { A _ { i _ { 1 } } h _ { \tau _ { 1 } } } \cdots e ^ { A _ { i _ { 1 } - n m } h _ { \tau _ { 1 } - n m } } \left(e^{A_{i_{\tau_{1}-n m-1}} h_{\tau_{1}-n m-1}} \cdots e^{A_{2} h_{1}} \mathcal{D}_{1}+\right.\right. \\
& \left.\left.\quad \cdots+\mathcal{D}_{\tau_{1}-n m-1}\right)+\Gamma_{A_{i_{\tau_{1}}}} \mathcal{V}_{1}+\mathcal{D}_{i_{\tau_{1}}}\right)
\end{aligned}
$$


Applying Lemma 2 once again, for almost all $h_{j}, j=\tau_{1}, \tau_{1}-m, \cdots, \tau_{1}-m n$, we have

$$
\begin{aligned}
& \operatorname{dim}\left(e^{A_{i_{1}} h_{\tau_{1}}} \cdots e^{A_{2} h_{1}} \mathcal{D}_{1}+\cdots+\mathcal{D}_{i_{\tau_{1}}}+\mathcal{V}_{1}\right) \\
& \geq \operatorname{dim}\left(e ^ { A _ { i _ { 1 } } h _ { \tau _ { 1 } } } \cdots e ^ { A _ { i _ { \tau _ { 1 } - n m } } h _ { \tau _ { 1 } - n m } } \left(e^{A_{\tau_{\tau_{1}-n m-1}} h_{\tau_{1}-n m-1}} \cdots e^{A_{2} h_{1}} \mathcal{D}_{1}\right.\right. \\
& \left.\left.+\cdots+\mathcal{D}_{\tau_{1}-n m-1}\right)+\Gamma_{A_{i_{1}}} \mathcal{V}_{1}+\mathcal{D}_{i_{\tau_{1}}}\right) \\
& \geq \operatorname{dim}\left(e ^ { A _ { i _ { \tau _ { 1 } - m } } h _ { \tau _ { 1 } - m } } \cdots e ^ { A _ { i _ { \tau _ { 1 } - n m } } h _ { \tau _ { 1 } - n m } } \left(e^{A_{\tau_{\tau_{1}}-n m-1} h_{\tau_{1}-n m-1}} \cdots e^{A_{2} h_{1}} \mathcal{D}_{1}\right.\right. \\
& \left.\left.+\cdots+\mathcal{D}_{\tau_{1}-n m-1}\right)+\Gamma_{A_{i_{1}}} \mathcal{V}_{1}+\mathcal{D}_{i_{\tau_{1}}}\right) \\
& \vdots \\
& \geq \operatorname{dim}\left(e ^ { A _ { i _ { 1 } - n m } h _ { \tau _ { 1 } - n m } } \left(e^{A_{\tau_{1}-n m-1} h_{\tau_{1}-n m-1}} \cdots e^{A_{2} h_{1}} \mathcal{D}_{1}+\cdots\right.\right. \\
& \left.\left.+\mathcal{D}_{\tau_{1}-n m-1}\right)+\Gamma_{A_{i_{\tau_{1}}}} \mathcal{V}_{1}+\mathcal{D}_{i_{\tau_{1}}}\right) \\
& =\operatorname{dim}\left(e^{A_{i_{1}-n m} h_{\tau_{1}-n m}} e^{A_{i_{\tau_{1}-n m-1}} h_{\tau_{1}-n m-1}} \cdots e^{A_{2} h_{1}} \mathcal{D}_{1}+\cdots\right. \\
& \left.+e^{A_{i_{\tau_{1}-n m}} h_{\tau_{1}-n m}} \mathcal{D}_{\tau_{1}-n m-1}+\mathcal{D}_{i_{\tau_{1}-n m}}+\Gamma_{A_{i_{1}}} \mathcal{V}_{1}\right)
\end{aligned}
$$

where the relationship $\mathcal{D}_{i_{\tau_{1}}}=\mathcal{D}_{i_{\tau_{1}-m n}}$ has been used.

Because each of (19) and (21) holds for almost all $h_{j}, j=\tau_{1}, \tau_{1}-m, \cdots, \tau_{1}-m n$, almost all choice of $h_{j}, j=\tau_{1}, \tau_{1}-m, \cdots, \tau_{1}-m n$ satisfies (19) and (21) simultaneously.

Continuing this process, we can prove that, for almost all $h_{j}, j=\tau_{1}-m n, \cdots, \tau_{1}-$ $m^{2} n+1$, we have

$$
\begin{aligned}
& \operatorname{dim}\left(e^{A_{i_{1}} h_{\tau_{1}}} \cdots e^{A_{2} h_{1}} \mathcal{D}_{1}+\cdots+\mathcal{D}_{i_{\tau_{1}}}+\mathcal{V}_{1}\right) \\
& \geq \operatorname{dim}\left(e^{A_{i_{1}-m n} h_{\tau_{1}-m n}} \cdots e^{A_{2} h_{1}} \mathcal{D}_{1}+\cdots+e^{A_{i_{\tau_{1}-m n}} h_{\tau_{1}-m n}} \mathcal{D}_{\tau_{1}-m n-1}\right. \\
& \left.+\mathcal{D}_{i_{\tau_{1}-m n}}+\Gamma_{A_{i_{1}}} \mathcal{V}_{1}\right) \\
& \geq \operatorname{dim}\left(e^{A_{i_{2}} h_{\tau_{2}}} \cdots e^{A_{2} h_{1}} \mathcal{D}_{1}+\cdots+\mathcal{D}_{i_{\tau_{2}}}+\Gamma_{A_{i_{1}}} \mathcal{V}_{1}+\cdots+\Gamma_{A_{i_{1}-m+1}} \mathcal{V}_{1}\right) \\
& =\operatorname{dim}\left(e^{A_{i_{2}} h_{\tau_{2}}} \cdots e^{A_{2} h_{1}} \mathcal{D}_{1}+\cdots+\mathcal{D}_{i_{\tau_{2}}}+\mathcal{V}_{2}\right)
\end{aligned}
$$

where $\tau_{2}=\tau_{1}-m^{2} n$.

Proceed the above reasonings, we finally have

$$
\begin{aligned}
& \operatorname{dim}\left(e^{A_{i_{l}} h_{l}} \cdots e^{A_{2} h_{1}} \mathcal{D}_{1}+\cdots+e^{A_{i_{l}} h_{l}} \mathcal{D}_{i_{l-1}}+\mathcal{D}_{i_{l}}\right) \\
& \geq \operatorname{dim}\left(e^{A_{i_{n}} h_{\tau_{n}}} \cdots e^{A_{2} h_{1}} \mathcal{D}_{1}+\cdots+\mathcal{D}_{i_{\tau_{n}}}+\mathcal{V}\right) \\
& \geq \operatorname{dim} \mathcal{V}
\end{aligned}
$$

where $\tau_{n}=l-\sum_{k=0}^{n-1} m(m n)^{k}$. 
Let $l \geq \sum_{k=0}^{n-1} m(m n)^{k}-1$, then from $(7)$ and $(22)$ it follows that

$$
\mathcal{R}_{s}\left(t_{f}\right)=\mathcal{V}
$$

which implies (14). $\diamond$

By Theorem 1, the controllable set is subspace $\mathcal{V}$. We thus refer to $\mathcal{V}$ as controllable subspace of system (1).

Theorem 2. For switched linear system (1), the controllable set is

$$
\mathcal{C}=\mathcal{V}
$$

The proof is completely parallel to that of Theorem 1 and hence is omitted.

Corollary 1. Both the controllable set and the reachable set are subspaces of the total space, and the two subspaces are always identical.

Corollary 2. For switched linear system (1), the following statements are equivalent

(i) The system is completely controllable;

(ii) The system is completely reachable; and

(iii) $\mathcal{V}=\Re^{n}$.

Due to Corollary 2, we can give an equivalent definition of controllability as follows.

Definition 7. System (1) is said to be (completely) controllable, if for any states $x_{0}$ and $x_{f}$, there exist a time instant $t_{f}>0$, a switching path $\sigma:\left[0, t_{f}\right] \rightarrow M$, and inputs $u_{k}:\left[0, t_{f}\right] \rightarrow \Re^{r_{k}}, k \in M$, such that $x\left(t_{f} ; 0, x_{0}, u, \sigma\right)=x_{f}$.

Remark 1. The controllable and reachable sets are invariant under re-arrangement of $A_{k}$ and $B_{k}$ for $k \in M$. That is, suppose both $j_{1}, \cdots, j_{m}$ and $l_{1}, \cdots, l_{m}$ are permutations of $1, \cdots, m$, then the controllable (reachable) set of system (1) coincide with that of the system given by

$$
\dot{x}(t)=\bar{A}_{\sigma} x(t)+\bar{B}_{\sigma} u_{\sigma}(t)
$$

where $\bar{A}_{k}=A_{j_{k}}$ and $\bar{B}_{k}=B_{l_{k}}$ for $k=1, \cdots, m$.

Remark 2. As noted in Example 1, although $\mathcal{R}$ and $\mathcal{C}$ coincide, $\mathcal{R}_{j}$ and $\mathcal{C}_{j}$ may differ from each other for certain $j$ s. This difference is due to incomplete switching which is a unique phenomenon of switched systems. 
Remark 3. For a non-switched linear system $(A, B)$, Corollary 2 degenerate to the well known geometric characterization for controllable subspace (Wonham 1979)

$$
\mathcal{C}=\mathcal{B}+A \mathcal{B}+\cdots+A^{n-1} \mathcal{B}
$$

\subsection{Switching control design}

By Theorems 1 and 2, any states in subspace $\mathcal{V}$ can transfer to each other in finite time. In this subsection, we study the following switching control design problem for switched system (1).

Switching Control Design Problem Given any two states $x_{0}$ and $x_{f}$ in the controllable subspace $\mathcal{V}$, find a switching path $\sigma$ and control input $u$ to steer the system from $x_{0}$ to $x_{f}$ in finite time.

Combining the proof of Theorem 1 and the geometric approach of linear systems (Wonham 1979), we can formulate a procedure to address this problem.

From the proof of Theorem 1, we can find a natural number $l$, positive real numbers $h_{1}, \cdots, h_{l}$, and an index sequence $i_{0}, \cdots, i_{l}$, such that equation (22) holds. This, together with (7), implies that

$$
e^{A_{i_{l}} h_{l}} \cdots e^{A_{2} h_{1}} \mathcal{D}_{1}+\cdots+e^{A_{i_{l}} h_{l}} \mathcal{D}_{i_{l-1}}+\mathcal{D}_{i_{l}}=\mathcal{V}
$$

Fix a positive real number $h_{0}$. Define the switching time sequence as

$$
t_{0}=0, t_{k}=t_{k-1}+h_{k-1}, \quad k=1, \cdots, l+1
$$

From the proof of Theorem 1.1 in Wonham (1979), for any $k \in M$ and $t>0$, we have

$$
\mathcal{D}_{k}=\operatorname{Im} W_{t}^{k}
$$

where

$$
W_{t}^{k}=\int_{0}^{t} e^{A_{k}(t-\tau)} B_{k} B_{k}^{T} e^{A_{k}^{T}(t-\tau)} d \tau
$$

Combining (26) with (28) leads to

$$
e^{A_{i_{l}} h_{l}} \cdots e^{A_{2} h_{1}} \operatorname{Im} W_{h_{0}}^{1}+\cdots+e^{A_{i_{l}} h_{l}} \operatorname{Im} W_{h_{l-1}}^{i_{l-1}}+\operatorname{Im} W_{h_{l}}^{i_{l}}=\mathcal{V}
$$


If we can formulate a control input $u$ satisfying the equation

$$
\begin{gathered}
x_{f}=x\left(t_{l+1}\right)=e^{A_{l} h_{l}} \cdots e^{A_{1} h_{0}} x_{0}+e^{A_{l} h_{l}} \cdots e^{A_{2} h_{1}} \int_{t_{0}}^{t_{1}} e^{A_{1}\left(t_{1}-\tau\right)} B_{1} u_{1}(\tau) d \tau \\
+\cdots+\int_{t_{l}}^{t_{l+1}} e^{A_{i_{l}}\left(t_{l+1}-\tau\right)} B_{i_{l}} u_{i_{l}}(\tau) d \tau
\end{gathered}
$$

then the switching control problem will be solved. To this end, consider the piecewise continuous control strategy

$$
u_{i_{k}}(t)=B_{i_{k}}^{T} e^{A_{i_{k}}^{T}\left(t_{k+1}-t\right)} a_{k+1}, t_{k} \leq t<t_{k+1}, k=0,1, \cdots, l
$$

where $a_{k} \in \Re^{n}, k=1, \cdots, l+1$ are vector variables to be determined.

Combining (30) with (31) gives

$$
\begin{gathered}
x_{f}-e^{A_{l} h_{l}} \cdots e^{A_{1} h_{0}} x_{0}=e^{A_{l} h_{l}} \cdots e^{A_{2} h_{1}} \int_{t_{0}}^{t_{1}} e^{A_{1}\left(t_{1}-\tau\right)} B_{1} B_{1}^{T} e^{A_{1}^{T}\left(t_{1}-t\right)} d \tau a_{1} \\
+\cdots+\int_{t_{l}}^{t_{l+1}} e^{A_{i_{l}}\left(t_{l+1}-\tau\right)} B_{i_{l}} B_{i_{l}}^{T} e^{A_{i_{l}}^{T}\left(t_{l+1}-t\right)} d \tau a_{l+1}
\end{gathered}
$$

This is equivalent to

$$
x_{f}-e^{A_{l} h_{l}} \cdots e^{A_{1} h_{0}} x_{0}=\left[e^{A_{i_{l}} h_{l}} \cdots e^{A_{2} h_{1}} W_{h_{0}}^{1}, \cdots, e^{A_{i_{l}} h_{l}} W_{h_{l-1}}^{i_{l-1}}, W_{h_{l}}^{i_{l}}\right] a
$$

where $a=\left[a_{1}^{T}, \cdots, a_{l+1}^{T}\right]^{T}$.

As $x_{f}-e^{A_{l} h_{l}} \cdots e^{A_{1} h_{0}} x_{0} \in \mathcal{V}$, it follows from (29) that linear equation (33) with unknown $a$ has at least has one solution. Solutions of linear equations (33) can be computed by symbolic or numerical softwares.

Suppose $a_{0}=\left[a_{0,1}^{T}, \cdots, a_{0, l+1}^{T}\right]^{T}$ is a solution of equation (33). Define the control inputs as

$$
u_{i_{k}}(t)=B_{i_{k}}^{T} e^{A_{i_{k}}^{T}\left(t_{k+1}-t\right)} a_{0, k+1}, t_{k} \leq t<t_{k+1}, k=0,1, \cdots, l
$$

and the switching path as

$$
\sigma(t)=i_{k}, \text { for } t \in\left[t_{k}, t_{k+1}\right), k=0,1, \cdots, l
$$

By the above reasonings, we have $x_{f}=x\left(t_{l+1} ; t_{0}, x_{0}, u, \sigma\right)$. That is, the piecewise continuous control input (34) and the switching path (35) constitute a solution for the switching control problem of switched system (1). 


\subsection{Computational issues}

As stated in Theorems 1 and 2, the controllable (reachable) set is subspace $\mathcal{V}$, which is defined recursively through $\left(A_{k}, B_{k}\right), k \in M$. The quantity relationship between them is

$$
\mathcal{V}=\sum_{i_{1}, \cdots, i_{n}=1, \cdots, m}^{j_{1}, \cdots, j_{n}=0,1, \cdots, n-1} A_{i_{n}}^{j_{n}} \cdots A_{i_{1}}^{j_{1}} \mathcal{B}_{i_{1}}
$$

That is, $\mathcal{V}$ is the summation of $(m n)^{n}$ items. It requires large computational effort to calculate this subspace if $m$ and $n$ are relatively large.

In this subsection, we provide a procedure to calculate $\mathcal{V}$ more efficiently.

Denote the nested subspaces as

$$
\begin{aligned}
& \mathcal{W}_{0}=\mathcal{B}_{1}+\cdots+\mathcal{B}_{m} \\
& \mathcal{W}_{j}=A_{1} \mathcal{W}_{j-1}+\cdots+A_{m} \mathcal{W}_{j-1}, j=1,2, \cdots
\end{aligned}
$$

Let $\mathcal{W}=\sum_{j=0}^{\infty} \mathcal{W}_{j}$. We then have

$$
\mathcal{W}_{0} \subset \mathcal{W}_{1} \subset \mathcal{W}_{2} \subset \cdots \subset \mathcal{W}, \text { and } \mathcal{V}=\mathcal{W}
$$

Note that if $\mathcal{W}_{j}=\mathcal{W}_{j+1}$ for some $j$, then $\mathcal{W}_{k}=\mathcal{W}_{j}$ for $k \geq j$ and further $\mathcal{W}_{j}=\mathcal{W}=\mathcal{V}$. This fact together with $\operatorname{dim} \mathcal{W} \leq n$ imply that $\mathcal{W}_{n-n_{0}}=\mathcal{W}=\mathcal{V}$, where $n_{0}=\operatorname{dim} \mathcal{W}_{0}$.

Denote

$$
\rho=\min \left\{k: \mathcal{W}_{k}=\mathcal{V}\right\} \leq n-n_{0}
$$

and

$$
n_{k}=\operatorname{dim} \mathcal{W}_{k}-\operatorname{dim} \mathcal{W}_{k-1}, \quad \mu_{k}=\sum_{j=0}^{k} n_{j}, k=1, \cdots, \rho
$$

A basis of $\mathcal{V}_{n}$ can be constructed according to the following procedure.

Firstly, choose a group of base vectors $\gamma_{1}, \cdots, \gamma_{s_{1}}$ in $\mathcal{B}_{1}$, expand them to $\gamma_{1}, \cdots, \gamma_{s_{1}}$, $\gamma_{s_{1}+1}, \cdots, \gamma_{s_{2}}$ which form a basis of $\mathcal{B}_{1}+\mathcal{B}_{2}$. Continuing this process, we can find a basis $\gamma_{1}, \cdots, \gamma_{n_{0}}$ of $\mathcal{W}_{0}$.

Secondly, because

$$
\begin{aligned}
\mathcal{W}_{1} & =\mathcal{W}_{0}+\operatorname{span}\left\{A_{k} \gamma_{j}, k=1, \cdots, m, j=1, \cdots, n_{0}\right\} \\
& =\operatorname{span}\left\{\gamma_{1}, \cdots, \gamma_{n_{0}}, A_{k} \gamma_{j}, k=1, \cdots, m, j=1, \cdots, n_{0}\right\}
\end{aligned}
$$


we can find a basis $\gamma_{1}, \cdots, \gamma_{n_{0}}, \gamma_{n_{0}+1}, \cdots, \gamma_{\mu_{1}}$ of $\mathcal{W}_{1}$ by searching the set

$$
\left\{\gamma_{1}, \cdots, \gamma_{n_{0}}, A_{k} \gamma_{j}, k=1, \cdots, m, j=1, \cdots, n_{0}\right\}
$$

from left to right.

Continuing the process, we can find a basis $\gamma_{1}, \cdots, \gamma_{n_{0}}, \cdots \gamma_{\mu_{l-1}+1}, \cdots, \gamma_{\mu_{l}}$ for $\mathcal{W}_{l}$. Because

$$
\begin{aligned}
\mathcal{W}_{l+1} & =\mathcal{W}_{l}+\operatorname{span}\left\{A_{j} \gamma_{k}, j=1, \cdots, m, k=\mu_{l-1}+1, \cdots, \mu_{l}\right\} \\
& =\operatorname{span}\left\{\gamma_{1}, \cdots, \gamma_{\mu_{k}}, A_{j} \gamma_{k}, j=1, \cdots, m, k=\mu_{l-1}+1, \cdots, \mu_{l}\right\}
\end{aligned}
$$

and by searching the set

$$
\left\{\gamma_{1}, \cdots, \cdots, \gamma_{\mu_{l}}, A_{j} \gamma_{k}, j=1, \cdots, m, k=\mu_{l-1}+1, \cdots, \mu_{l}\right\}
$$

from left to right for linearly independent column vectors, we can find a basis $\gamma_{1}, \cdots, \gamma_{n_{0}}$, $\cdots \gamma_{\mu_{l-1}+1}, \cdots, \gamma_{\mu_{l}}, \gamma_{\mu_{l}+1}, \cdots, \gamma_{\mu_{l+1}}$ for $\mathcal{W}_{l+1}$.

Finally, we have $\mathcal{V}=\operatorname{span}\left\{\gamma_{1}, \cdots, \gamma_{n_{0}}, \cdots \gamma_{\mu_{\rho-1}+1}, \cdots, \gamma_{\mu_{\rho}}\right\}$. It involves not more than $\sum_{k \in M} r_{k}+m \mu_{\rho-1}$ column vectors in the procedure, which is only a small fraction of the original quantity, $(m n)^{n}$.

Remark 4. From the above analysis, a basis for $\mathcal{V}$ is of the form

$$
\left\{b_{1}, A_{i_{1,1}} b_{1}, A_{i_{k_{1}, 1}} \cdots A_{i_{1,1}} b_{1}, \cdots, \cdots, b_{n_{0}}, A_{i_{1, n_{0}}} b_{1}, A_{i_{k_{n_{0}}, n_{0}}} \cdots A_{i_{1, n_{0}}} b_{n_{0}}\right\}
$$

where $b_{j} \in \mathcal{W}_{0}, k_{j} \geq 0, j=1, \cdots, n_{0}, 1 \leq i_{l, j} \leq m, l=1, \cdots, k_{j}, j=1, \cdots, n_{0}$. Because the number of vectors in (38) is not more than $n$, there are at most $n$ different subsystems whose parameters appear in (38). That is to say, for controllability and reachability issues, we may assume $m \leq n$ without loss of generality.

\section{Observability and determinability}

In the above analysis, reference is made to reachability and controllability only. It should be noticed that the observability and determinability counterparts can be addresses dualistically. In this section, we outline the relevant concepts and the corresponding criteria.

Consider a switched linear control system with outputs given by

$$
\begin{aligned}
\dot{x}(t) & =A_{\sigma} x(t)+B_{\sigma} u(t) \\
y(t) & =C_{\sigma} x(t)
\end{aligned}
$$


where $x(t) \in \Re^{n}, u(t) \in \Re^{p}$ and $y(t) \in \Re^{q}$ are the states, inputs and outputs, respectively, $\sigma: \Re \rightarrow M=\{1,2, \cdots, m\}$ is the switching path to be designed, and $A_{k}, B_{k}, C_{k}$, $k \in M$ are constant matrices of compatible dimension.

Definition 8. The switched linear system (39) is (completely) observable, if there exist a time $t_{1}>0$ and a switching path $\sigma:\left[0, t_{1}\right] \rightarrow M$, such that state $x(0)$ can be determined from knowledge of the output $y(t), t \in\left[0, t_{1}\right]$ and the input $u(t), t \in\left[0, t_{1}\right]$.

Definition 9. The switched linear system (39) is (completely) determinable, if there exist an time $t_{1}<0$ and a switching path $\sigma:\left[t_{1}, 0\right] \rightarrow M$, such that state $x(0)$ can be determined from knowledge of the output $y(t), t \in\left[t_{1}, 0\right]$ and the input $u(t), t \in\left[t_{1}, 0\right]$.

In view of Theorems 1 and 2 for reachability and controllability, the following criteria are readily obtained for observability and determinability by using the principle of duality.

Theorem 3. For switched linear system (39), the following statements are equivalent

(i) The system is completely observable;

(ii) The system is completely determinable; and

(iii) $\mathcal{O}=\Re^{n}$

where subspace $\mathcal{O}$ is defined recursively by

$$
\begin{aligned}
\mathcal{O}_{1} & =\operatorname{Im} C_{1}^{T}+\cdots+\operatorname{Im} C_{m}^{T} \\
\mathcal{O}_{j+1} & =\Gamma_{A_{1}^{T}} \mathcal{O}_{j}+\cdots+\Gamma_{A_{m}^{T}} \mathcal{O}_{j}, \quad j=1,2, \cdots
\end{aligned}
$$

\section{An illustrative example}

Example 2. Consider the switched systems given by

$$
A_{1}=0, B_{1}=e_{1}, A_{j}=e_{j} e_{j-1}^{T}, B_{j}=0, j=2, \cdots, m, m \leq n
$$

where $e_{j}, 1 \leq j \leq n$ is the unit column vector with the ith entry equal to one.

To compute the controllable subspace $\mathcal{V}$, we follow the procedure presented in Section 4.3 . 
It can be readily seen that

$$
\mathcal{W}_{0}=\operatorname{span}\left\{e_{1}\right\}
$$

By searching the independent vectors in

$$
\mathcal{W}_{1}=\operatorname{span}\left\{e_{1}, A_{j} e_{1}, j=1, \cdots, m\right\}
$$

we obtain that

$$
\mathcal{W}_{1}=\operatorname{span}\left\{e_{1}, A_{2} e_{1}\right\}=\operatorname{span}\left\{e_{1}, e_{2}\right\}
$$

Continue this process, we have

$$
\mathcal{W}_{k}=\operatorname{span}\left\{e_{1}, \cdots, e_{k}, A_{j} e_{k}, j=1, \cdots, m\right\}=\operatorname{span}\left\{e_{1}, \cdots, e_{k+1}\right\}
$$

for $k=2, \cdots, m-1$, and

$$
\mathcal{W}_{m}=\operatorname{span}\left\{e_{1}, \cdots, e_{m}, A_{j} e_{m}, j=1, \cdots, m\right\}=\operatorname{span}\left\{e_{1}, \cdots, e_{m}\right\}=\mathcal{W}_{m-1}
$$

Thus $\mathcal{V}=\mathcal{W}=\mathcal{W}_{m-1}$. According to Theorems 1 and 2, the controllable (reachable) set is

$$
\mathcal{R}=\mathcal{C}=\operatorname{span}\left\{e_{1}, \cdots, e_{m}\right\}
$$

which is an $m$-dimensional subspace. If $m=n$, then the switched system is controllable and reachable.

Now let us address the switching control problem for system (41). Following the procedure outlined in Section 4.2, we consider the periodic switching index sequence and piecewise continuous inputs.

Let us choose the switching time sequence to be

$$
t_{0}=0, t_{1}=1, t_{2}=2, \cdots
$$

Accordingly, $h_{k}=h=1$ for $k=0,1, \cdots$. Simple calculation gives

$$
e^{A_{1} h}=I_{n}, e^{A_{j} h}=I_{n}+A_{j}, j=2, \cdots, m
$$

where $I_{n}$ is the $n$th order identity matrix.

Let $l=m l_{0}$ with $l_{0}$ to be determined. Under the periodic switching index sequence (15), we can compute that

$$
\begin{aligned}
& \operatorname{dim}\left(e^{A_{i_{l}} h} \cdots e^{A_{2} h} \mathcal{D}_{1}+\cdots+e^{A_{i_{l}} h} \mathcal{D}_{i_{l-1}}+\mathcal{D}_{i_{l}}\right) \\
& =\operatorname{dim}\left(e^{A_{i_{l}} h} \cdots e^{A_{2} h} \mathcal{D}_{1}+e^{A_{i_{l}} h} \cdots e^{A_{i_{l-m+1}} h} \cdots e^{A_{i_{l-m}} h} \mathcal{D}_{1}+\cdots+\mathcal{D}_{1}\right) \\
& =\operatorname{dim}\left(Q^{l_{0}} \mathcal{B}_{1}+Q^{l_{0}-1} \mathcal{B}_{1}+\cdots+\mathcal{B}_{1}\right)
\end{aligned}
$$


where

$$
Q=e^{A_{m} h} e^{A_{m-1} h} \cdots e^{A_{2} h}=I_{n}+A_{2}+\cdots+A_{m}
$$

It can be verified that vectors $B_{1}, Q B_{1}, \cdots, Q^{m-1} B_{1}$ are linearly independent, and

$$
\mathcal{V}=\operatorname{span}\left\{B_{1}, Q B_{1}, \cdots, Q^{m-1} B_{1}\right\}
$$

Accordingly, we choose that $l_{0}=m-1$.

Simple calculation gives

$$
W_{h}^{1}=e_{1} e_{1}^{T}, \quad W_{t}^{k}=0, k=2, \cdots, m
$$

For any given states $x_{0}$ and $x_{f}$ in $\mathcal{V}$, consider the equation

$$
x_{f}-Q^{m-1} x_{0}=\left[Q^{m-1} W_{h}^{1}, \cdots, Q W_{h}^{1}, W_{h}^{1}\right] a
$$

Let $P$ denote the sub-matrix of $\left[Q^{m-1} B_{1}, \cdots, Q B_{1}, B_{1}\right]$ consisting the first $m$ rows. It is clear that $P$ is nonsingular. Denote

$$
a_{0}=\left[P^{-1}, 0\right]\left(x_{f}-Q^{m-1} x_{0}\right)
$$

The solutions of equation (44) are given by

$$
a=\left[a_{0}(1), *, \cdots, *, a_{0}(2), *, \cdots, *, \cdots, a_{0}(m), *, \cdots, *\right]^{T}
$$

where $a_{0}(j)$ denotes the $j$ th entry of vector $a_{0}$, and the symbol '*' stands for any real numbers.

The corresponding piecewise continuous input is

$$
u_{1}(t)=a_{0}(j+1), t_{m j} \leq t<t_{m j+1}, j=0, \cdots, m-1
$$

The switching index sequence (15) and control strategy (45) will steer the system from original $x_{0}$ at $t=0$ to the target $x_{f}$ at $t=m(m-1)+1$.

The above switching control scheme involves $m(m-1)$ times of switching to transfer between two arbitrarily given states in the controllable subspace. This number can be reduced, if we use aperiodic switching index sequence instead of the periodic one. For example, let us consider the switching index sequence

$$
i_{0}=1, i_{1}=2, i_{2}=1, i_{3}=3, i_{4}=2, i_{5}=1, \cdots, i_{k-m+1}=m, \cdots, i_{k}=1
$$


where $k=\frac{(m-1)(m+2)}{2}$. Under the switching time sequence (42), we have

$$
\begin{aligned}
& \operatorname{dim}\left(e^{A_{i_{k}} h} \cdots e^{A_{i_{1}} h} \mathcal{D}_{i_{0}}+\cdots+e^{A_{i_{k}} h} \mathcal{D}_{i_{k-1}}+\mathcal{D}_{i_{k}}\right) \\
& =\operatorname{dim}\left(e^{A_{i_{k}} h} \cdots e^{A_{i_{1}} h} \mathcal{D}_{1}+e^{A_{i_{k}} h} \cdots e^{A_{i_{k-m}}{ }^{h}} \mathcal{D}_{1}+\cdots+\mathcal{D}_{1}\right) \\
& =\operatorname{dim}\left(Q_{m-1} \mathcal{B}_{1}+Q_{m-2} \mathcal{B}_{1}+\cdots+Q_{1} \mathcal{B}_{1}+\mathcal{B}_{1}\right)
\end{aligned}
$$

where matrices $Q_{j}, j=1, \cdots, m$ are defined recursively as

$$
Q_{1}=e^{A_{2}} \cdots e^{A_{m}}, \quad Q_{j}=e^{A_{2}} \cdots e^{A_{m+1-j}} Q_{j-1}, j=2, \cdots, m-1
$$

It can be verified that vectors $B_{1}, Q_{1} B_{1}, \cdots, Q_{m-1} B_{1}$ are linearly independent, and

$$
\mathcal{V}=\operatorname{span}\left\{B_{1}, Q_{1} B_{1}, \cdots, Q_{m-1} B_{1}\right\}
$$

Accordingly, a piecewise control input can be obtained by solving the following equation

$$
x_{f}-Q_{m-1} x_{0}=\left[Q_{m-1} W_{h}^{1}, \cdots, Q_{1} W_{h}^{1}, W_{h}^{1}\right] a
$$

for any given initial state $x_{0}$ and target state $x_{f}$.

An interesting question arise naturally: Is $\frac{(m-1)(m+2)}{2}$ the minimal switching number for system (41)? Or equivalently, is this number can be further reduced by other switching index sequences? We could not provide a definite answer yet, though we incline the positive answer.

\section{Conclusion}

In this paper, detailed controllability and reachability analyse have been carried out for switched linear control systems. It has been proven that, both the controllable and reachable sets are subspaces of the total space, and the two sets always coincide with each other. The controllable (reachable) set is exactly the minimal $A_{k^{-}}$invariant subspace for $k \in M$ which contains $\sum_{k \in M} \mathcal{B}_{k}$. Criteria for observability and determinability have also been obtained by duality. These results generalize Wonham's geometric characterizations to switched systems.

A closely related interesting problem is controlling the switched linear systems with minimum number of switching. It seems that more rigorous rank estimation for exponential matrix should be developed to address this problem.

\section{ACKNOWLEDGEMENT}

The authors would like to thank the anonymous reviewers for their constructive and insightful comments for further improving the quality of this work. 


\section{References}

Blondel, V. D., \& Tsitsiklis, J. N. (1999). Complexity of stability and controllability of elementary hybrid systems. Automatica, 35(3), 479-489.

Branicky, M. S. (1998). Multiple Lyapunov functions and other analysis tools for switched and hybrid systems. IEEE Transactions on Automatic Control, 43(4), $475-482$.

Brockett, R. W. (1983). Asymptotic stability and feedback stabilization. In R. W. Brockett et al, Differential Geometric Control Theory (pp. 181-191). Boston: Birkhauser.

Caines, P. E., \& Wei, Y.-J. (1998). Hierarchical hybrid control systems: a lattice theoretic formulation. IEEE Transactions on Automatic Control, 43(4), 501-508.

Chase, C., Serrano, J., \& Ramadge, P. J. (1993). Periodicity and chaos from switched flow systems: contrasting examples of discretely controlled continuous systems. IEEE Transactions on Automatic Control, 38(1), 70-83.

Conner, L. T. Jr., \& Stanford, D. P. (1987). The structure of controllable set for multimodal systems. Linear Algebra and its Applications, 95(2), 171-180.

Dayawansa, W. P., \& Martin, C. F. (1999). A converse Lyapunov theorem for a class of dynamical systems which undergo switching. IEEE Transactions on Automatic Control 44(4), 751-760.

Drager, L.D., Foote, R.L., Martin, C.F., \& Wolfer, J. (1989). Controllability of linear systems, differential geometry curves in Grassmannians and generalized Grassmannians, and Riccati equations. Acta Applicandae Mathematicae, 16(3), 281-317.

Ezzine, J., \& Haddad, A. H. (1989). Controllability and observability of hybrid systems. International Journal of Control, 49(6), 2045-2055.

Kaplan, W. (1966). Introduction to Analytic Functions. Reading: Addison-Wesley.

Kolmanovsky, I., \& McClamroch, N. H. (1995). Developments in nonholonomic control problems. IEEE Control Systems, 15(6), 20-36.

Kolmanovsky, I., \& McClamroch, N. H. (1996). Hybrid feedback laws for a class of cascade nonlinear control systems. IEEE Transactions on Automatic Control, 41(9), $1271-1282$. 
Leonessa, A., Haddad, W. M., \& Chellaboina, V. (2001). Nonlinear system stabilization via hierarchical switching control. IEEE Transactions on Automatic Control, 46(1), 17-28.

Li, Z. G., Wen, C. Y., \& Soh, Y. C. (2001). Switched controllers and their applications in bilinear systems. Automatica, 37(3), 477-481.

Liberzon, D., \& Morse, A. S. (1999). Basic problems in stability and design of switched systems. IEEE Control Systems, 19(5), 59-70.

Loparo, K. A., Aslanis, J. T., \& IIajek, O. (1987). Analysis of switching linear systems in the plain, part 2, global behavior of trajectories, controllability and attainability. Journal of Optimization Theory and Applications, 52(3), 395-427.

Morse, A. S.(1996). Supervisory control of families of linear set-point controllers- Part 1:, exact matching. IEEE Transactions on Automatic Control, 41(10), 1413-1431.

Narendra, K. S., \& Balakrishnan, J. (1997). Adaptive control using multiple models. IEEE Transactions on Automatic Control, 42(2), 171-187.

Savkin, A. V., Skafidas, E., \& Evans, R. J. (1999). Robust output feedback stabilizability via controller switching. Automatica, 35(1), 69-74.

Sira-Ramirez, H. (1991). Nonlinear P-I controller design for switch mode dc-to-dc power converters. IEEE Transactions and Circuits and Systems, 38(4), 410-417.

Stanford, D. P., \& Conner, L. T. Jr. (1980). Controllability and stabilizability in multipair systems. SIAM Journal on Control and Optimization, 18(5), 488-497.

Sun, Z., \& Zheng, D. Z. (2001). On reachability and stabilization of switched linear control systems. IEEE Transactions on Automatic Control, 46(2), 291-295.

Szigeti, F. (1992). A differential-algebraic condition for controllability and observability of time varying linear systems. In Proceedings of 31st Conference on Decision and Control (pp. 3088-3090).

Wicks, M. A., Peleties, P., \& DeCarlo, R. A. (1998). Switched controller synthesis for the quadratic stabilization of a pair of unstable linear systems. European Journal of Control, 4(2), 140-147. 
Williams, S. M., \& Hoft, R. G. (1991). Adaptive frequency domain control of PPM switched power line conditioner. IEEE Transactions on Power Electronics, 6(4), 665-670.

Wonham, W. M. (1979). Linear Multivariable Control - A Geometric Approach. Berlin: Spinger-Verlag.

Xu, X., \& Antsaklis, P. J. (1999). On the reachability of a class of second-order switched systems. Technical report, ISIS-99-003, University of Notre Dame.

Ye, H., Michel, A. N., \& Hou, L. (1998). Stability theory for hybrid dynamical systems. IEEE Transactions on Automatic Control, 43(4), 461-474. 